\title{
The clinical value of detecting circulating tumour cells in the peripheral blood of nasopharyngeal carcinoma patients
}

\author{
ZHUO CHEN $^{1 *}, \mathrm{LU} \mathrm{XU}^{1 *}, \mathrm{XINHUA} \mathrm{XU}^{1}$ and CHEN YUAN ${ }^{2}$ \\ ${ }^{1}$ Department of Oncology, The First College of Clinical Medical Science, China Three Gorges University \\ and Yichang Central People's Hospital; ${ }^{2}$ Department of Oncology, The First College of Clinical \\ Medical Science, China Three Gorges University, Yichang, Hubei 443003, P.R. China
}

Received October 7, 2017; Accepted February 13, 2018

DOI: $10.3892 / \mathrm{ol} .2018 .8155$

\begin{abstract}
The aim of the present study as to analyse the associations between circulating tumour cells (CTCs) and the clinical parameters of nasopharyngeal carcinoma (NPC). Peripheral blood $(7.5 \mathrm{ml})$ from 68 first-diagnosed NPC patients was collected to detect and identify CTCs by cluster of differentiation (CD) 45 immunomagnetic separation. Immunofluorescent staining of cytokeratin-18, CD45 and DAPI, and fluorescence in situ hybridization were combined with the centromere of chromosome 8 (CEP8) probe method to analyse the associations between CTCs and clinical parameters. One-year follow-up of the NPC patients who received standardized treatment was also performed to analyse the associations between CTCs, tumour development and the treatment effect. The detection rate of CTCs in the 68 NPC patients was $98.5 \%$ and the positive rate of CTCs was $60.3 \%$. The positive rates of CTCs in the I-III and IV stage patients were 51.1 and $78.3 \%$, respectively; the rate was $90.0 \%$ in the $\mathrm{M}_{1}$ stage and $55.2 \%$ in the $\mathrm{M}_{0}$ stage. The differences were statistically significant $(\mathrm{P}<0.05)$. The mean CTC counts were $3.86 \pm 2.36$ and $5.70 \pm 2.91$ in the $\mathrm{M}_{0}$ and $\mathrm{M}_{1}$ stages, respectively, which was significantly different $(\mathrm{P}=0.031)$. The 12-month follow-up record suggested tumour progression for 17 patients, and the one-year progress free survival rate was $74.6 \%$. Among the CTC-positive stages III-IV patients, the disease progression rate of the patients who had received treatment including chemotherapy/intensity-modulated radiation therapy (IMRT) was $83.3 \%$, which was higher than that of the patients who received treatment including
\end{abstract}

Correspondence to: Professor Xinhua $\mathrm{Xu}$, Department of Oncology, The First College of Clinical Medical Science, China Three Gorges University and Yichang Central People's Hospital, 183 Yiling Road, Yichang, Hubei 443003, P.R. China

E-mail: xuxinhua@medmail.com.cn

${ }^{*}$ Contributed equally

Key words: nasopharyngeal carcinoma, circulating tumour cells, clinical pathological features, treatment
chemotherapy/IMRT/chemotherapy, and the difference was statistically significant $(\mathrm{P}<0.05)$. The results of the present study suggested that CTCs were closely associated with the stages of NPC. The later clinical stages may have higher CTC-positive rates for NPC. Treatment with chemotherapy/IMRT/chemotherapy may be more effective for CTC-positive patients in stages III-IV than the use of chemotherapy/IMRT.

\section{Introduction}

Nasopharyngeal carcinoma (NPC) is a common malignant head and neck cancer that mainly occurs in China and southeast Asia (1); intensity-modulated radiation therapy (IMRT) is a major therapeutic approach for NPC. Although ongoing improvement of precise radiation therapy has resulted in a 5-year survival rate of $70 \%$ for NPC patients in China, $30-40 \%$ of the patients have a risk of tumour recurrence and metastasis after the first diagnosis. For patients with a high risk of tumour recurrence and metastasis, radiotherapy plus chemotherapy is a highly recommended therapy. However, there remains significant room to study the pathological and clinical features of the NPC patients who enjoy better therapy effects from chemotherapy.

Recent studies have shown that CTCs, which could be dispersed into circulation system by primary and metastatic cancers, are closely correlated with recurrence and metastasis of several tumour types, including breast cancer (2), lung cancer (3), gastric cancer (4), head and neck cancers (5-7), as well as resistance to anti-tumour treatment. Additionally, it is of significant clinical guiding value in auxiliary diagnosis, therapeutic evaluation, recurrence and metastasis monitoring and prognostic assessment of tumour patients. Wu et al (8) also found that the decrease of CTCs was related with better therapeutic efficacy in NPC patients and the CTC might be a predictive factor for clinical outcomes via continuously detecting CTC count in a treatment (8). In our study, we also aim to analyse the correlation between CTCs and the clinical features of NPC.

\section{Materials and methods}

Ethical approval. The study was approved by the Ethics Committee of the First College of Clinical Medical Science, 
China Three Gorges University and Yichang Central People's Hospital (Yichang, China), and all patients provided written informed consent. All procedures performed in studies involving human participants were in accordance with the ethical standards of the institutional and/or national research committee and with the 1964 Helsinki declaration and its later amendments or comparable ethical standards.

Subjects and samples. This study included 68 NPC patients and 10 healthy individuals (undergoing routine physical examination) from Yichang Central People's Hospital, Gezhouba Central Hospital, the Second People's Hospital of Yichang and Renhe Hospital during the period from March, 2014 to February, 2015. The NPC patients included 38 males and 30 females, whose ages were from 27 to 72 years with an average of 50 years old. According to The 2008 TMN Staging System for Nasopharyngeal Carcinoma (NPC), 5 were identified as stage I, 12 as stage II, 28 as stage III, 13 as stage IVa and 10 as stage IVb. All the patients had been diagnosed by histopathological diagnosis or fine-needle aspiration biopsy with clear TMN staging. Those with other tumours were excluded. All participants lacked active infection and malfunction or failure of important internal organs, including the liver, kidney, heart, etc. CTCs of all samples were detected and identified prior to providing the patients with any treatment.

The patients in stages I-II received IMRT as the first therapy (prescription dose 95\%PGTVnx=72.6 Gy/ $2.2 \mathrm{~Gy} / \mathrm{fx} 33 \mathrm{~F}, 95 \% \mathrm{PGTVnd}=72.6 \mathrm{~Gy} / 2.2 \mathrm{~Gy} / \mathrm{fx} 33 \mathrm{~F}$, 95\%PTV1=62.04 Gy/1.88 Gy/fx33 F, 95\%PTV2=56.76 Gy/ $1.72 \mathrm{~Gy} / \mathrm{fx} 33 \mathrm{~F})$. The patients in stages III-IV received a 2-period treatment of inductive chemotherapy (cisplatin at $25 \mathrm{mg} / \mathrm{m}^{2}, \mathrm{~d} 1-\mathrm{d} 3$; 5-fluoride of $600 \mathrm{mg} / \mathrm{m}^{2}, \mathrm{~d} 1-\mathrm{d} 5$ ) first. Then, they received a 2-period treatment of IMRT at the same dose as above. One month after completion of IMRT, 31 patients received a 4-period chemotherapy (DF regimen). The peripheral blood of 10 healthy volunteers was extracted to serve as a control group. All patients underwent CT and MRI examination one month after the treatment, and they underwent re-examination every 2-3 months. The results of a 12-month follow-up study were recorded.

Enrichment and identification of CTCs. The samples of $7.5 \mathrm{ml}$ of peripheral blood were incubated with anti-cluster of differentiation (CD)45 magnetic nanoparticles and collected for subsequent CTC enrichment and detection; then, CTCs were identified through the combination of CD45 and CK18 with the FISH-centromere of chromosome 8 (CEP8) probe method. Enriched cells were made into cell smears. In the following steps, CEP8 probe was denatured at $75^{\circ} \mathrm{C}$ for 10 mins before it was added to the slides. Afterwards, the slides were kept in a hybridization oven for $2 \mathrm{~h}$. After hybridization, an antibody mixture of CD45 and CK18 was added to the slides with one-hour incubation. In the final stage, DAPI was added and the CTCs were analyzed with a fluorescence microscope (by immunostaining, not western blotting and FACS).

Statistical analysis. Data were analyzed using statistic analysing software SPSS 18.0. The $\chi^{2}$ test, t-test, and Kaplan-Meier analysis were performed to analyse the data.

\section{Results}

Classification and identification of CTCs. According to the theoretical criteria, the cells under the microscope were classified as eitherCK18 ${ }^{-}$or $\mathrm{CK} 18^{+}, \mathrm{CD}_{4} 5^{+}$or CD45-, and CEP8 $>2$ (the number of hybridization signals for CEP8>2) or CEP8=2. Only three patterns are detected: $\mathrm{CD} 45^{+} / \mathrm{CK} 18^{-} / \mathrm{CEP} 8=2$ (A pattern), CD45 $/ \mathrm{CK} 18 \%$ CEP8=2 (B pattern), and CD45\%CK18\%/CEP8>2 (C pattern) (Figs. 1-3). The A pattern represented white blood cells, the $\mathrm{C}$ pattern indicated CTCs, and the B pattern indicated suspicious CTCs, which were not included in the CTC count. Cells of the $\mathrm{C}$ pattern and the $\mathrm{B}+\mathrm{C}$ pattern were detected in 67 of the 68 samples with a CTC detection rate of $98.5 \%$. According to the criterion that CTCs $\geq 3 / 7.5 \mathrm{ml}$ was considered positive [technically supported by the Kindstar Global Co., Ltd. (Wuhan, China) and the standard set based on a large number of pre-clinical screening], the positive rate of CTCs was $60.3 \%$ (41/68). No C-pattern cell was detected in the peripheral blood of the healthy individuals.

CTC count in clinicopathologic parameters. Based on the CTC evaluation criteria described above, the number of CTCs detected in the peripheral blood of the 68 NPC patients ranged from 0 to 12 cells $/ 7.5 \mathrm{ml}$ with a median of $4.13 \pm 2.51 / 7.5 \mathrm{ml}$. The CTC number increased with an increase in the pathological stages with mean counts of $3.71 \pm 2.33$ (stages I-III) and $4.96 \pm 2.69$ (stage IV), respectively. However, the difference was not statistically significant $(\mathrm{P}=0.052)$. The mean count of CTCs was $3.86 \pm 2.36$ in the $\mathrm{M}_{0}$ stage, and it was $5.70 \pm 2.91$ in the $\mathrm{M}_{1}$ stage, which was significantly different $(\mathrm{P}=0.031$; Table I).

CTC-positive rates and clinicopathologic parameters. The positive rates of CTCs in the peripheral blood of the patients in stages I-III and stage IV were $51.1 \%$ (23/45) and $78.3 \%$ $(18 / 23)$; the difference was statistically significant $(\mathrm{P}=0.038)$. The positive rate of CTCs was $90.0 \%(9 / 10)$ in the $M_{1}$ stage and $55.2 \%(32 / 58)$ in the $\mathrm{M}_{0}$ stage; the difference was also statistically significant $(\mathrm{P}=0.043)$. Among the different groups of sexs, ages, pathological types, $\mathrm{T}$ stages and $\mathrm{N}$ stages, the CTC-positive rates were not statistically significant $(\mathrm{P}>0.05$; Table II).

The prognostic value of CTC in NPC. According to the data of a 12-month follow-up, 17 patients were found to have disease progression, and the one-year progress free survival (PFS) rate was $74.6 \%$. Among the cases with disease progression, there was 1 patient in stages I-II (5.9\%) and 16 in stages III-IV (32.0\%). The disease-progression rates in CTC-positive and CTC-negative patients were 34.1 and $11.5 \%$, respectively, and the difference was significantly different $(\mathrm{P}=0.047$; Table III). Among the 50 patients in stages III-IV, 31 received treatment with inductive chemotherapy/IMRT/chemotherapy (A treatment) and 19 received inductive chemotherapy/IMRT (B treatment). The disease-progression rates were $16.1 \%$ with the A treatment and $57.9 \%$ with the B treatment. For 35 CTC-positive stage III-IV cases, the disease-progression rate was $83.3 \%$ with $\mathrm{B}$ treatment and $17.4 \%$ with A treatment. There was a significant difference between the two groups ( $\mathrm{P}<0.05$; Fig. 4). 


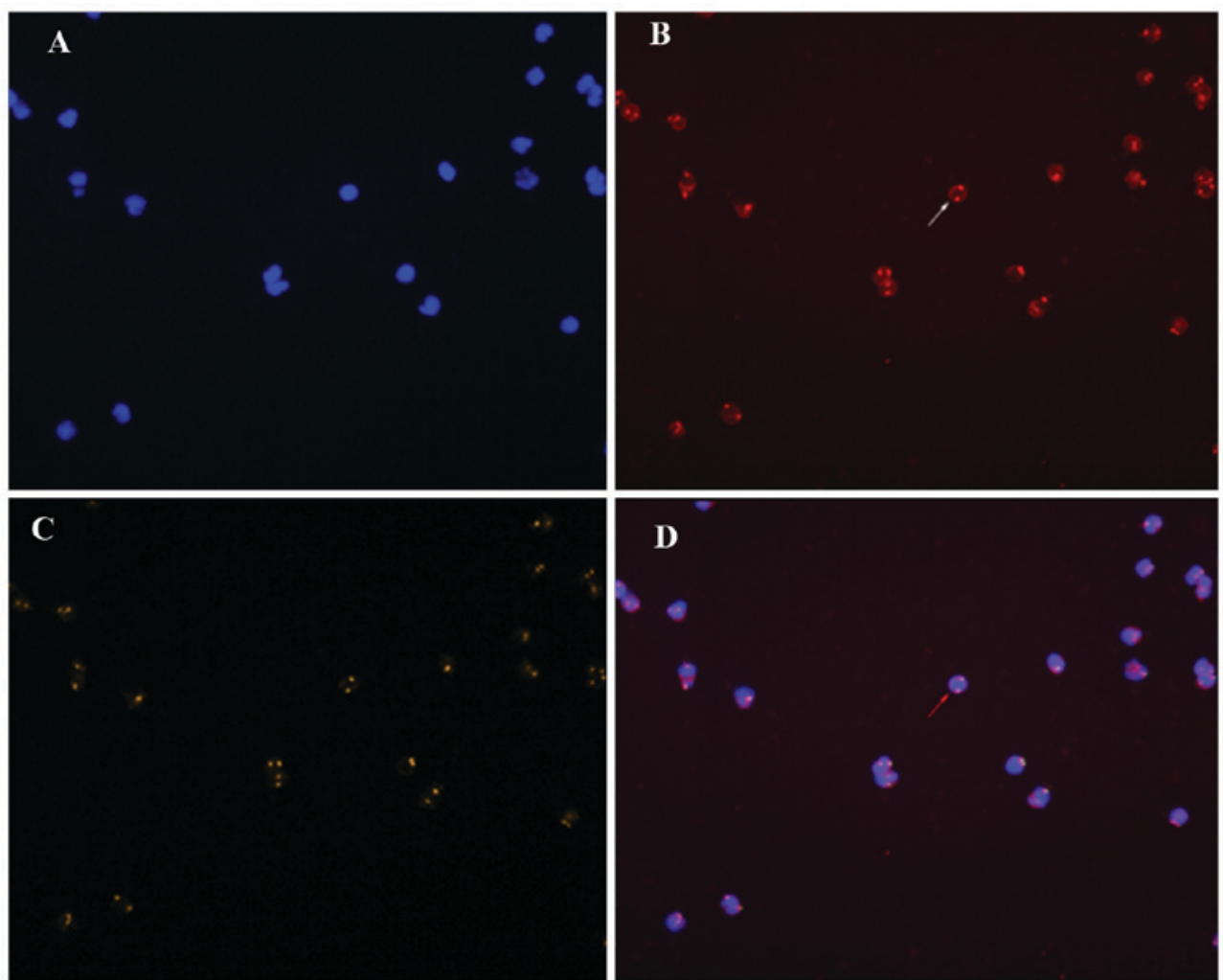

Figure 1. Exemplificative images cells detected through combining CK18, CD45, DAPI and fluorescence in situ. CK18 $/$ CD45 $/$ CEP8 $=2$ cell considered as white blood cell. The all samples of CK18 were negative and were not displayed. The arrows indicate the representative cells. (A) DAPI blue staining, (B) CD45 red staining, (C) CEP8 orange staining and (D) the merged image (magnification, x40). CK, cytokeratin; CD, cluster of differentiation; CEP8, centromere of chromosome 8.

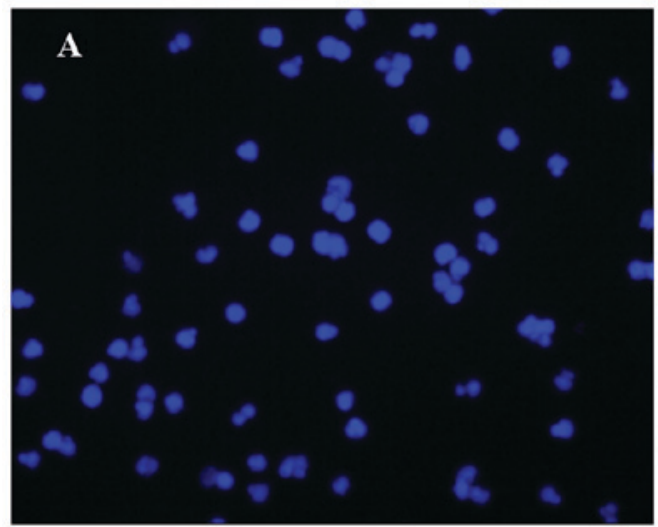

\section{B}
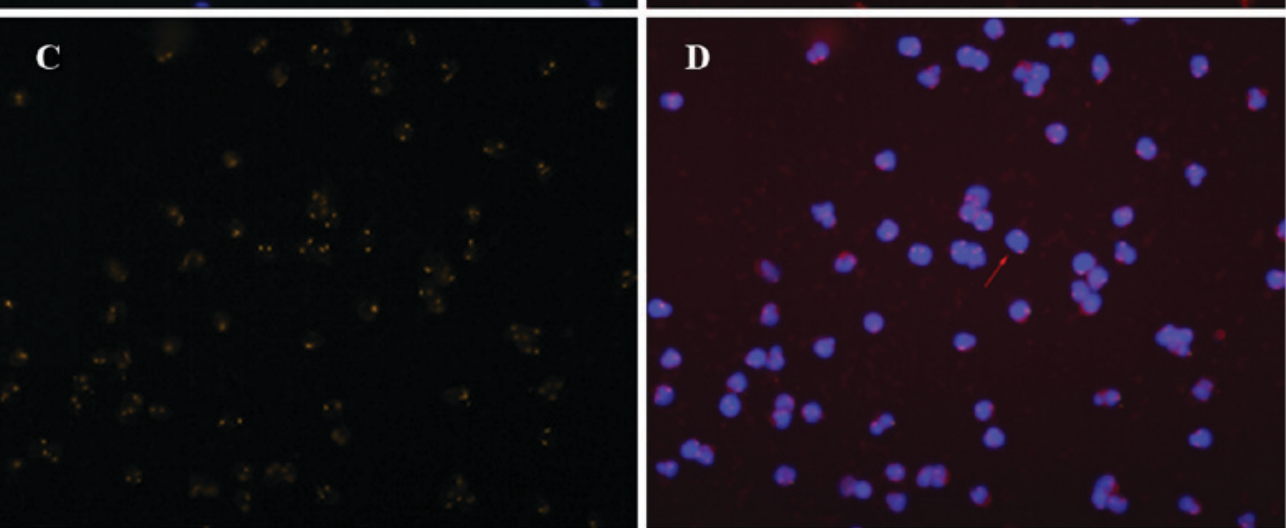

Figure 2. Exemplificative images cells detected through combining CK18, CD45, DAPI and fluorescence in situ. CK18 $/ \mathrm{CD} 45 / \mathrm{CEP} 8=2$ cell considered as suspicious CTC. The all samples of CK18 were negative and were not displayed. The arrows indicate the representative cells. (A) DAPI blue staining, (B) CD45 red staining, (C) CEP8 orange staining and (D) the merged image (magnification, $\mathrm{x} 40$ ). CK, cytokeratin; CD, cluster of differentiation; CEP8, centromere of chromosome 8. 
Table I. Circulating tumor cell numbers and nasopharyngeal carcinoma clinical features.

\begin{tabular}{lccr}
\hline Clinical feature & Range $(\mathrm{n})$ & $\begin{array}{c}\text { Circulating tumor cell numbers } \\
\text { (mean } \pm \text { standard deviation) }\end{array}$ & P-value \\
\hline Tumor stage & $0-9$ & $3.71 \pm 2.33$ & 0.052 \\
I-III & $1-12$ & $4.96 \pm 2.69$ & 0.108 \\
IV & $0-9$ & $3.26 \pm 2.60$ & \\
Primary tumor & $1-7$ & $3.91 \pm 1.90$ & 0.795 \\
T1 & $1-9$ & $4.60 \pm 2.41$ & \\
T2 & $1-12$ & $5.45 \pm 3.17$ & \\
T3 & & & \\
T4 & $0-12$ & $3.54 \pm 3.20$ & \\
Lymph nodes & $1-8$ & $4.11 \pm 2.42$ & \\
N0 & $1-9$ & $4.42 \pm 2.41$ & \\
N1 & $1-9$ & $4.23 \pm 2.20$ & \\
N2 & & $3.86 \pm 2.36$ & \\
N3 & $0-9$ & $5.70 \pm 2.91$ & \\
Metastasis & $1-12$ & & \\
M0 & & & \\
M1 & &
\end{tabular}

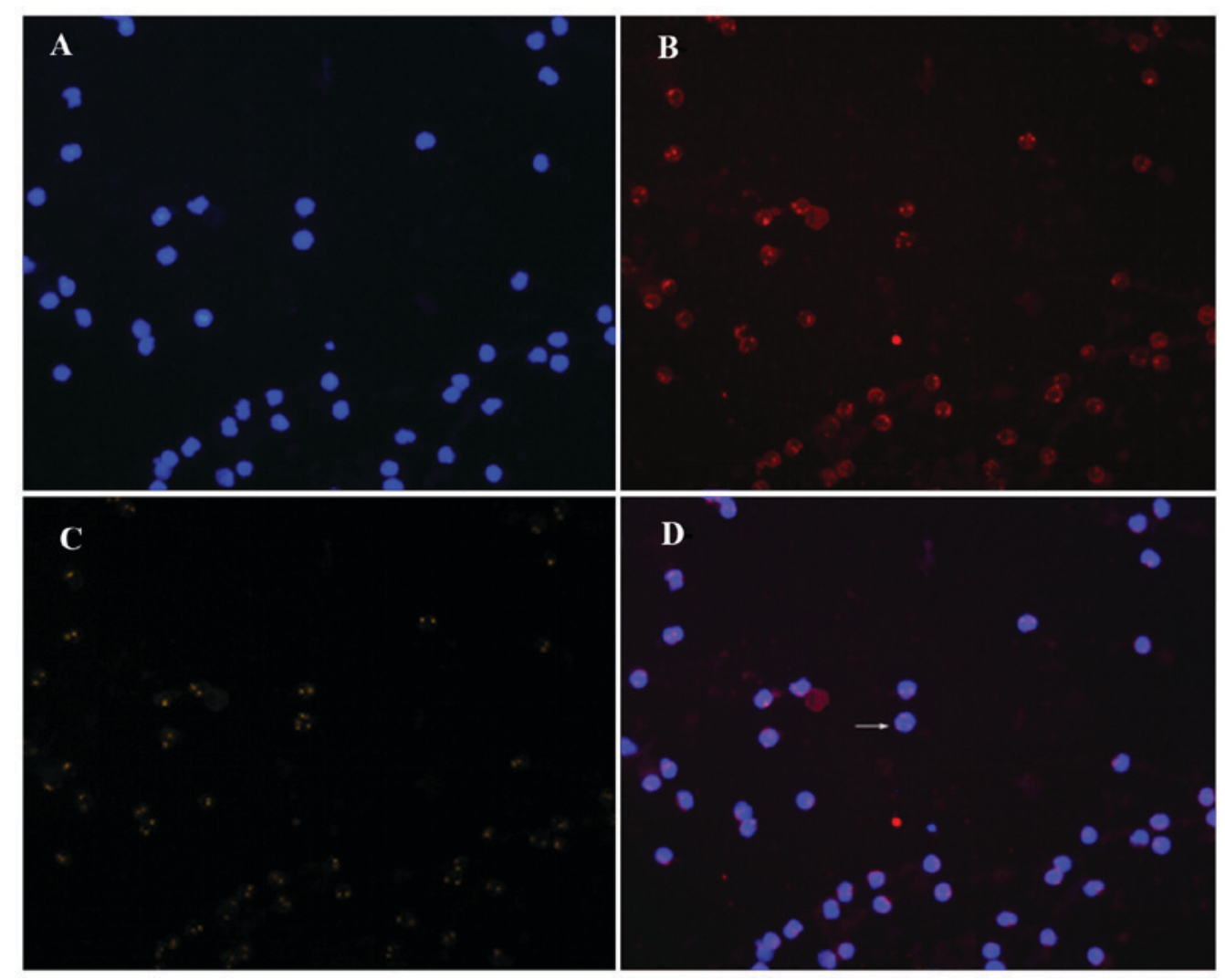

Figure 3. Exemplificative images cells detected through combining CK18, CD45, DAPI and fluorescence in situ. CK18/CD45/CEP8>2 cell considered as CTC. The all samples of CK18 were negative and were not displayed. The arrows indicate the representative cells. (A) DAPI blue staining, (B) CD45 red staining, (C) CEP8 orange staining and (D) the merged image (magnification, $\mathrm{x} 40$ ). CK, cytokeratin; CD, cluster of differentiation; CEP8, centromere of chromosome 8 .

\section{Discussion}

The methods for CTC detection remain inconsistent with each other; the difference in the choice of tumour markers and testing methods contributes to this. Comparatively, a relatively mature method for CTC detection involves the use of EpCAM and $\mathrm{CK}$ expression as indicators of tumour presence. However, some studies have confirmed that the epithelial-mesenchymal 
Table II. Circulating tumor cell positive rate and nasopharyngeal carcinoma clinical features.

\begin{tabular}{|c|c|c|c|c|c|}
\hline Clinical feature & Cases (n) & $\begin{array}{l}\text { CTC positive/ } \\
\text { negative }(n)\end{array}$ & CTC positive rate & $\chi^{2}$ & $\mathrm{P}$-value \\
\hline Sex & & & & 1.087 & 0.328 \\
\hline Male & 38 & $25 / 13$ & 65.8 & & \\
\hline Female & 30 & $16 / 14$ & 53.3 & & \\
\hline Age (years) & & & & 0.911 & 0.455 \\
\hline$>50$ & 38 & $21 / 17$ & 55.3 & & \\
\hline$\leq 50$ & 30 & $20 / 10$ & 66.7 & & \\
\hline Histological type & & & & 0.230 & 0.780 \\
\hline Non-keratinizing carcinoma & 50 & $31 / 19$ & 62.0 & & \\
\hline $\begin{array}{l}\text { Keratinized squamous } \\
\text { cell carcinoma }\end{array}$ & 18 & $10 / 8$ & 55.6 & & \\
\hline Primary tumor & & & & 6.373 & 0.096 \\
\hline $\mathrm{T} 1$ & 19 & $7 / 12$ & 36.8 & & \\
\hline $\mathrm{T} 2$ & 23 & $15 / 8$ & 65.2 & & \\
\hline $\mathrm{T} 3$ & 15 & $11 / 4$ & 73.3 & & \\
\hline $\mathrm{T} 4$ & 11 & $8 / 3$ & 72.7 & & \\
\hline Lymph nodes & & & & 4.666 & 0.194 \\
\hline N0 & 13 & $5 / 8$ & 38.5 & & \\
\hline N1 & 18 & $10 / 8$ & 55.6 & & \\
\hline $\mathrm{N} 2$ & 24 & $16 / 8$ & 66.7 & & \\
\hline N3 & 13 & $10 / 3$ & 76.9 & & \\
\hline Metastasis & & & & 4.322 & 0.043 \\
\hline M0 & 58 & $32 / 26$ & 55.2 & & \\
\hline M1 & 10 & $9 / 1$ & 90.0 & & \\
\hline Tumor stage & & & & 4.686 & 0.038 \\
\hline I-III & 45 & $23 / 22$ & 51.1 & & \\
\hline IV & 23 & $18 / 5$ & 78.3 & & \\
\hline
\end{tabular}

CTC, circulating tumor cell.

transition (EMT) of CTCs may reduce the expression of epidermal protein, which can reduce the efficiency of the testing method. Krebs et al (9) found the diagnostic rate of lung CTC is $30 \%$ in a test based on EpCAM and CK expression. So many new technologies to detect CTCs are applied in recent years $(6,7,10)$. unfortunately, there are a few researches to compare the detection efficiency of the various new technologies. In this study, CTCs are identified by combining immunofluorescent staining of CK18 and CD45 and FISH with the (CEP8) probe method followed by subtractive enrichment of CD45. The subtractive enrichment approach is independent of down-regulation or loss of EpCAM expression and increases the diagnostic rate $(10,11)$. CTCs are CD45(-) non-haematopoietic cells, and the chromosomal instability of tumour cells causes polyploidization of FISH-CEP8. CD45/CK18 $/ \mathrm{CEP} 8 \geq 2$ and CD45/CK18\%/CEP8>2 are considered as indicators of CTCs because of CK positivity or hyperdiploid. Our collaborating laboratory discovered that the CK18-positive rate was 5-6\% with this detection method based on testing of hundreds of other cancer samples. In this study, only three patterns were detected, $\mathrm{CD} 45^{+} / \mathrm{CK} 18^{-} / \mathrm{CEP} 8=2$ (A pattern), CD $45 \%$ CK $18 \% / C E P 8=2$ (B pattern) and CD45/CK18-/CEP8>2
(C pattern). The C pattern suggested detection of CTCs, and the $\mathrm{B}$ pattern indicated expressive attributes consistent with suspicious CTCs, which were not included in the CTC count.

The detection rate of CTCs was $98.5 \%$ in the peripheral blood of NPC patients, and the positive rate of CTC was $60.3 \%$. No CTCs were detected in the peripheral blood of healthy individuals. No CK-positive NPC patients were found in this study, as supported the occurrence of CTC EMT. It also suggested that further studies on different physiological states of CTCs could promote a better understanding of the mechanism of tumour development and provide a theoretical basis for the diagnosis and treatment of tumours. Additionally, the cells of B pattern might be white cells that have not been infected with CD45 antibodies or they might be CK-negative diploid tumour cells.

The positive rates of CTCs were 51.1 and $78.3 \%$ in the peripheral blood of NPC patients with stages I-III and IV, respectively. The positive rate of CTCs was closely correlated to the result of clinical staging; a later stage reveals a higher positive rate of CTCs. The positive rate of CTCs in the $\mathrm{M}_{1}$ stage was higher than that in the $\mathrm{M}_{0}$ stage. A higher CTC-positive 
Table III. One-year disease progression in nasopharyngeal carcinoma.

\begin{tabular}{|c|c|c|c|c|c|c|}
\hline Clinical feature & Cases (n) & Progression & Free-progress & Progress ratio & $\chi^{2}$ & P-value \\
\hline Tumor stage & & & & & 4.570 & 0.050 \\
\hline I-II & 17 & 1 & 16 & 5.9 & & \\
\hline III-IV & 50 & 16 & 34 & 32.0 & & \\
\hline CTC number & & & & & 4.295 & 0.047 \\
\hline $\mathrm{CTC} \geq 3$ & 41 & 14 & 27 & 34.1 & & \\
\hline CTC $<3$ & 26 & 3 & 23 & 11.5 & & \\
\hline Primary tumor & & & & & 3.359 & 0.364 \\
\hline $\mathrm{T} 1$ & 19 & 3 & 16 & 15.8 & & \\
\hline $\mathrm{T} 2$ & 22 & 5 & 17 & 22.7 & & \\
\hline $\mathrm{T} 3$ & 15 & 4 & 11 & 26.7 & & \\
\hline $\mathrm{T} 4$ & 11 & 5 & 6 & 45.4 & & \\
\hline Lymph nodes & & & & & 4.370 & 0.125 \\
\hline N0-1 & 31 & 5 & 26 & 16.1 & & \\
\hline $\mathrm{N} 2$ & 23 & 6 & 17 & 26.1 & & \\
\hline N3 & 13 & 6 & 7 & 46.2 & & \\
\hline Metastasis & & & & & 3.765 & 0.107 \\
\hline M0 & 57 & 12 & 45 & 21.1 & & \\
\hline M1 & 10 & 5 & 5 & 50.0 & & \\
\hline Histological type & & & & & 0.985 & 0.527 \\
\hline $\begin{array}{l}\text { Keratinized squamous } \\
\text { cell carcinoma }\end{array}$ & 18 & 3 & 15 & 16.7 & & \\
\hline Non-keratinizing carcinoma & 49 & 14 & 35 & 28.6 & & \\
\hline Sex & & & & & 0.048 & 0.827 \\
\hline Male & 37 & 9 & 28 & 24.3 & & \\
\hline Female & 30 & 8 & 22 & 26.7 & & \\
\hline Age (years) & & & & & 0.614 & 0.574 \\
\hline$>50$ & 37 & 8 & 29 & 21.6 & & \\
\hline$\leq 50$ & 30 & 9 & 21 & 30.0 & & \\
\hline
\end{tabular}

CTC, circulating tumor cell.

rate could suggest a higher probability of tumour recurrence. These were consistent with a poor prognosis and high risk of metastasis for advanced patients.

Based on the study finding, it can be hypothesized that the release of CTCs is a dynamic pathological process. The level of released CTCs is affected by the tumour burden, and an increase in the tumour burden might increase the number of CTCs. It was found that the difference between the CTC-positive rates of the $\mathrm{T}$ and $\mathrm{N}$ stages was not statistically significant. One reason might be that the size of the primary tumour is a factor that modulates the release of CTCs. Meanwhile, compared to other solid tumours, the NPC location is relatively limited, limiting the growth of primary tumours.

Considering tumour metastasis is a complex multi-step process and positive detection of CTCs does not guarantee the formation of metastases, we also analyzed the numbers of CTCs in the peripheral blood of NPC patients. The mean count of CTCs in the $M_{0}$ stage was lower than that in the $M_{1}$ stage, due to the CTC numbers was really low, more samples were needed if we analyzed the statistical significance.
CTCs are killed via the body's immune response and their own metabolism $(12,13)$, resulting in the survival of some CTCs that contribute to further development of specific conditions. The more CTCs detected, the more CTCs might survive until the final stage, increasing the risk of distant metastasis; this was consistent with the study result.

Based on a 12-month follow-up survey, we found the one-year progression free survival (PFS) rate was $74.6 \%$, and the disease-progression rate in CTC-positive cases was higher than that in CTC-negative cases. A higher CTC-positive rate might mean a poorer prognosis, which coincided with the hypothesis raised above. At present, the therapeutic schedule for NPC patients with stages I-II was relatively identical and consisted of IMRT. For advanced (III-IV) NPC patients, the combination of IMRT and chemotherapy is highly recommended. However, the specific patients who would benefit more from the therapy is still not very clear. In 50 cases of stages III-IV, the disease-progression rate of patients who received treatment with inductive chemotherapy/IMRT (B treatment) was higher than that who received 


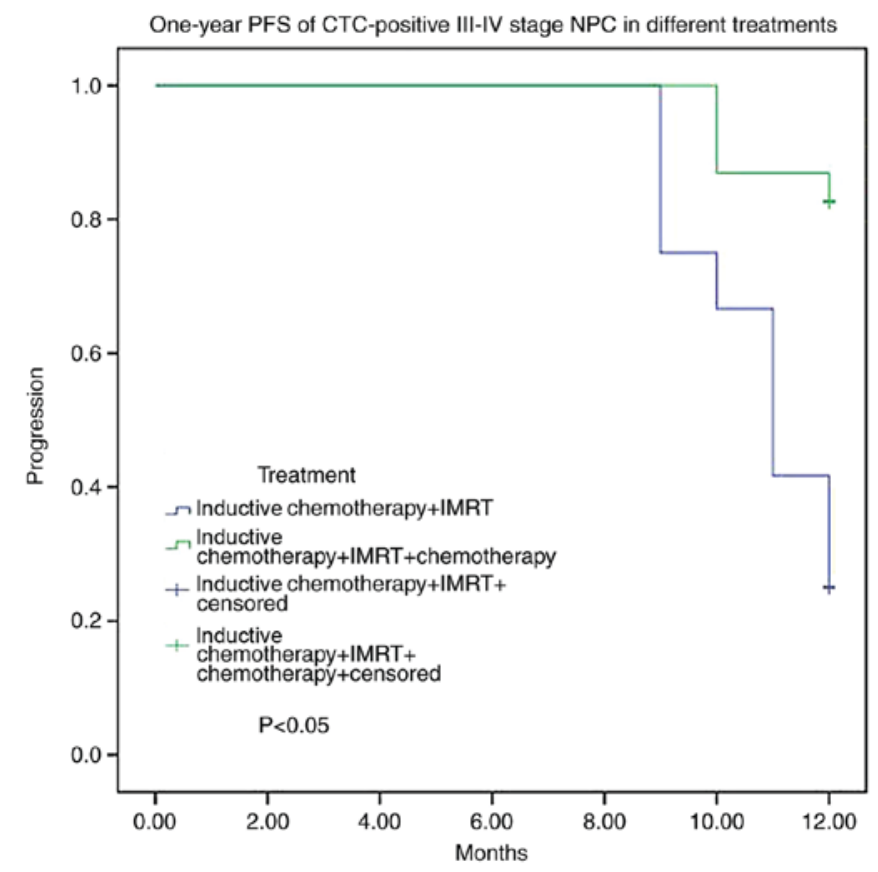

Figure 4. One-year PFS of CTC-positive III-IV stage NPC in different treatments. CTC-positive patients of the III-IV stages who received different treatments (inductive chemotherapy/IMRT or inductive chemotherapy/IMRT/chemotherapy) were followed-up and the one-year PFS was evaluated. The PFS were analyzed by Kaplan-Meier analyses. The difference was statistically significant $(\mathrm{P}<0.05)$. PFS, progress free survival; CTC, circulating tumour cells; IMRT, intensity-modulated radiation therapy.

inductive chemotherapy/IMRT/chemotherapy (A treatment). Additionally, the A treatment might be more effective. For CTC-positive cases of stages III-IV, the disease-progression rate was higher with $\mathrm{B}$ treatment than with $\mathrm{A}$ treatment. For advanced NPC patients, especially CTC-positive cases, A treatment can be considered as the first choice.

To conclude, we found that the positive rate of CTCs was correlated with the clinical stages of NPC patients and distant tumour metastasis. Treatment with inductive chemotherapy + IMRT + chemotherapy might be more effective for CTC-positive patients with NPC stages III-IV. Unfortunately, there are still many insufficient. It has been documented that EB virus is associated with NPC, we do not analyze the relationship of CTCs and EBV in our study. With the advance of technology, newer or higher effective detection techniques may be discovered. Besides, the number of samples employed in this study is comparatively low, and the follow-up period is somewhat short. As a result, we could not comprehensively demonstrate the relationship between CTCs and the prognosis of NPC patients. Because a larger sample size could strengthen the conclusions, we will continue to enrol more cases and follow-up the prognosis.

\section{Acknowledgements}

Not applicable.

\section{Funding}

The present study was funded by Natural Science Foundation of Hubei, China (grant no. 2014CFB312).

\section{Availability of data and materials}

All data generated or analyzed during this study are included in this published article.

\section{Authors' contributions}

ZC and LX made substantial contributions to the study design and the acquisition, analysis and interpretation of the data. CY took part in the analysis of the data and $\mathrm{XX}$ contributed to the study conception and agreed to be accountable for all aspects of the work.

\section{Ethics approval and consent to participate}

The study was approved by the Ethics Committee of the First College of Clinical Medical Science, China Three Gorges University and Yichang Central People's Hospital (Yichang, China), and all patients provided written informed consent. All procedures performed in studies involving human participants were in accordance with the ethical standards of the institutional and/or national research committee, and with the 1964 Helsinki Declaration and its later amendments or comparable ethical standards.

\section{Consent for publication}

Patients provided written informed consent for the publication of their data.

\section{Competing interests}

The authors declare that they have no competing interests.

\section{References}

1. Ferlay J, Soerjomataram I, Dikshit R, Eser S, Mathers C, Rebelo M, Parkin DM, Forman D and Bray F: Cancer incidence and mortality worldwide: Sources, methods and major patterns in GLOBOCAN 2012. Int J Cancer 136: E359-E386, 2015.

2. Rack B, Schindlbeck C, Jückstock J, Andergassen U, Hepp P, Zwingers T, Friedl TW, Lorenz R, Tesch H, Fasching PA, et al: Circulating tumor cells predict survival in early average-to-high risk breast cancer patients. J Natl Cancer Inst 106: pii: dju066, 2014.

3. Chen YY and Xu GB: Effect of circulating tumor cells combined with negative enrichment and CD45-FISH identification in diagnosis, therapy monitoring and prognosis of primary lung cancer. Med Oncol 31: 240, 2014

4. Kulasinghe A, Perry C, Jovanovic L, Nelson C and Punyadeera C: Circulating tumour cells in metastatic head and neck cancers. Int J Cancer 136: 2515-2523, 2015.

5. Ning N, Zhan T, Zhang Y, Chen Q, Feng F, Yang Z, Liu Z, Xu D, Wang F, Guo Y, et al: Improvement of specific detection of circulating tumor cells using combined CD45 staining and fluorescence in situ hybridization. Clin Chim Acta 433: 69-75, 2014.

6. Si Y, Lan G, Deng Z, Wang Y, Lu Y, Qin Y, Huang B, Yang Y, Weng J, Han X, et al: Distribution and clinical significance of circulating tumor cells in nasopharyngeal carcinoma. Jpn J Clin Oncol 46: 622-630, 2016

7. He C, Huang X, Su X, Tang T, Zhang X, Ma J, Guo X and Lv X: The association between circulating tumor cells and Epstein-Barr virus activation in patients with nasopharyngeal carcinoma. Cancer Biol Ther 18: 888-894, 2017.

8. Wu Y, Qian Y, Peng J, Wei X, Yuan Z, Wei S and Hu D: Clinical evaluation of circulating tumor cells in locally advanced nasopharyngeal carcinoma: A prospective study. J Clin Oncol 35: e17523, 2017. 
9. Krebs MG, Sloane R,Priest L,Lancashire L,Hou JM, Greystoke A, Ward TH, Ferraldeschi R, Hughes A, Clack G, et al: Evaluation and prognostic significance of circulating tumor cells in patients with non-small cell lung cancer. J Clin Oncol 29: 1556-1563, 2011.

10. Zhang Y, Wang F, Ning N, Chen Q, Yang Z, Guo Y, Xu D, Zhang D, Zhan T and Cui W: Patterns of circulating tumor cells identified by CEP8, CK and CD45 in pancreatic cancer. Int J Cancer 136: 1228-1233, 2015.

11. Okabe H, Tsunoda S, Hosogi H, Hisamori S, Tanaka E, Tanaka S and Sakai Y: Circulating tumor cells as an independent predictor of survival in advanced gastric cancer. Ann Surg Oncol 22: 3954-3961, 2015.

12. Kim HJ, Choi GS, Park JS, Park S, Kawai K and Watanabe T: Clinical significance of thrombocytosis before preoperative chemoradiotherapy in rectal cancer: Predicting pathologic tumor response and oncologic outcome. Ann Surg Oncol 22: 513-519, 2015.
13. Santos MF, Mannam VK, Craft BS, Puneky LV, Sheehan NT, Lewis RE and Cruse JM: Comparative analysis of innate immune system function in metastatic breast, colorectal, and prostate cancer patients with circulating tumor cells. Exp Mol Pathol 96: 367-374, 2014. 